\title{
Las revistas científicas desde sus nexos con la responsabilidad social. Reflexiones
}

\author{
Manuel Paulino LinARES HerRera \\ Universidad de La Habana \\ mplinares106@gmail.com
}

\begin{abstract}
Resumen:
El presente debate investigativo al que convoca este artículo, pretende evidenciar la importancia de las revistas científicas en el ámbito de la comunicación de resultados de investigación, sustentándose en la responsabilidad social como un análisis específico y necesario. Se precisa como en la actualidad la concepción divulgativa en las revistas científicas es éticamente responsable. Las revistas científicas como objetivo de análisis demuestra en el debate, como se comprometen con la responsabilidad social que les atañe, como canal comunicativo y vehículo en cohesión con los lineamientos científicos, tecnológicos y medioambientales que estipula la sociedad para su desarrollo, siendo un producto comunicativo intangible, incide la capacidad de progreso de la sociedad.
\end{abstract}

Palabras clave: Comunicación científica; revistas científicas; responsabilidad social; comunicación de resultados; ciencia; tecnología y sociedad.

\section{Scientific from its ties to social responsibility journals. Reflections}

\begin{abstract}
:
This research debate convenes this article aims to show the importance of scientific journals in the field of communication of research results, premised on the social responsibility as a specific and necessary analysis. It is accurate as at present the informative concept in scientific journals is ethically responsible. Journals objective analysis as demonstrated in the discussion are committed to social responsibility that affects them, as communication channel and cohesion vehicle with scientific, technological and environmental guidelines that society provides for its development, being an intangible product communicative , affects the ability of the progress of society.
\end{abstract}

Key Words: Science communication; scientific journals; social responsibility; communication of results; science, technology and society.

\section{Referencia normalizada:}

Linares Herrera, M. P. (2014): Las revistas científicas desde sus nexos con la responsabilidad social. Reflexiones. Historia y Comunicación Social. Vol. 19. Núm. Especial Marzo. Págs. 807-818.

Sumario: 1. Introducción. En las ciencias: las revistas científicas como vehículo de comunicación. 2. Fundamento de la propuesta reflexiva. 3. La comunicación y su visibilidad, prismas de la responsabilidad social. 4. La comunicación en las revistas científica condicionada al interés social. 5. Las revistas: un potencial canal de comunicación científica. 6. Como conclusiones: 


\section{Introducción en las ciencias: las revistas científicas como vehículos de comu- nicación}

Cuando asumimos actitudes, compromisos y estilos de comportamiento en función de la sociedad, y desde organizaciones decisorias, sobre todo asumiendo un rol ante los diferentes contextos sociales, estamos poniendo en práctica a la necesaria responsabilidad social que le debe corresponder a todo sujeto en función social, como vía hacia el desarrollo y como fundamento social.

Núñez Jover; Montalvo A.; Figaredo C; (2008, p. 152), plantean en particular que la responsabilidad social en el caso de los profesionales de las ciencias...

....se ha convertido en uno de los principales factores de desarrollo del potencial científico, lo cual ha redundado en el rendimiento práctico de las ciencias, ya que el hecho mismo de hacer ciencia y tecnología implica, necesariamente, asumir un grado de responsabilidad específico

En la actualidad la percepción por la responsabilidad social, ha ido evolucionando desde los contextos socio-culturales, políticos y económicos, siendo hoy un concepto normativo, de obligatoria implementación y específico desde sus ámbitos, donde la sociedad augura por el desarrollo y su sostenibilidad. Las acciones provocadas por el impacto socio-cultural, ambiental, ecológico y tecnológico que se van propiciando, generan actividades y tomas de decisiones profesionales y especializadas desde organizaciones decisorias, para el desarrollo de la sociedad y su mejora en la calidad de vida.

Desde las ciencias, la responsabilidad social toma todos los pilares de la sostenibilidad, esta no es más que planificar con vista a un futuro a largo alcance, donde estén implicadas las generaciones futuras de la sociedad y se tenga en cuenta el preservar a la naturaleza, sus recursos, al ser humano y a todos los demás entornos que le conforman. Son condiciones determinantes e inherentes para el desarrollo de la sociedad. La conceptualización de las ciencias, independientemente de la disciplina que anuncie, es una premisa que fundamenta toda una amplia investigación sobre problemáticas en torno a temas de ciencia, tecnología y sociedad (CTS), sumidos estos estudios en la responsabilidad, como uno de los ente que justifica el desarrollo social, desde esta arista se perfila la reflexión de este artículo, en torno a las revistas científicas.

Las ciencias se contextualizan ante el desarrollo que va aconteciendo en las comunidades científicas, es un proceso que transita. "La vieja ciencia se va transfiriendo a la nueva a través de un proceso acumulativo", Núñez Jover; Montalvo A.; Figaredo C; (2008, p. 119-128). La ciencia ha evolucionando, su filosofía, su concepciones en el método científico a aplicar, por lo que puntualiza el autor que "la ciencia es una actividad profesional institucionalizada que supone educación prolongada, internacionalización de valores, creencias, desarrollo de estilos de pensamiento y actuación. La ciencia es todo una cultura y así debe ser estudiada".

La responsabilidad social se detiene para un análisis específico, cuando la valoramos desde el científico y su papel ante la comunicación de sus resultados, se precisa 
que su función es la de preservar que estas investigaciones no puedan traer consecuencias nefastas para la sociedad, que la espera será para la aplicación de procedimientos éticos que devengan en procesos importantes y decisorias para el desarrollo de la sociedad y la humanidad en su conjunto. Las instituciones empresariales desde su objeto social y sus parámetros en gestión del conocimiento deben incidir por la actuación responsable del científico, asevera Gutiérrez-Cuevas, C. (2001).

Otra visión del proceder en la responsabilidad social lo define, la evolución epistemológica de las CTS, la cual se ha contextualizado por los diversos matices socio-culturales y desde el tema de estudio: las revistas científicas, la responsabilidad social, se asume de forma sectorizada de acuerdo a sus ámbitos, que son las comunidades y disciplinas científicas que las conforman (ciencias exactas, naturales, humanidades, médicas, técnicas, y otras diferentes) en función de las acciones que devengan las investigaciones que se comunican.

Un escenario base en la comunicación de resultados científicos se manifiesta en la universidad como parte determinante de los procesos en pos del desarrollo, por lo que le deviene una importante misión en los procedimientos de comunicación científica.

Retomando sobre la literatura existente en torno al tema de la Responsabilidad Social, esta investigación se apoya en el ya realizado estudio de Quesada, Gaete (2011) quien argumenta sobre diversas percepciones en torno al tema. Desde el enfoque cronológico de este término Carroll, (1979) sugiere convertir a este término en una fuerte línea de investigación, la falta de consenso en aquel entonces lo impedía, los dogmas y la falta de consentimientos también influían.

Los planteamientos de Garriga y Melé (2004), y los de Secchi (2007), según los autores de la investigación de referencia: Quesada, Gaete (2011), trazaron pautas y permitieron perfilar varios enfoques sobre el concepto de responsabilidad social, sobre todo desde una perspectiva institucional focalizados en el accionar hacia las actividades sociales y sus demandas, con el correspondiente tratamiento ético que procuran estos procedimientos.

Estos enfoques han permitido realizar profundos estudios, que se han sectorizados y encaminados hacia un desarrollo sostenible, dígase con miras al futuro de las nuevas generaciones, al desarrollo a largo plazo, desde las posibilidades y recursos disponibles como premisa de trabajo, comprometida desde las organizaciones decisorias y sus diferentes ámbitos.

Las representaciones en la gestión de resultados científicos desde sus nexos con la responsabilidad social, la conforman: el universitario y el institucional, también referidos como de organizaciones, estas conforman las directrices del quehacer científico, en el sector universitario a través de políticas de formación y, de estrategias éticas de comportamientos y aprendizajes; a su vez desde las organizaciones o instituciones se perfilan desde el cómo orientar sus premisa, a nivel de implementación, con acciones participativas, solidarias y de abertura a la interrelación con las problemáticas de la 
sociedad, donde las revistas científicas a través de sus diferentes canales de divulgación, cumplen su rol comunicativo.

Desde la percepción del conocimiento las ciencias se implican con la responsabilidad social, se manifiesta durante el proceso del individuo para conocer, y prepararse profesionalmente y enfrentar las tareas de las ciencias, su metodología científica, con objetividad y el caracterizado rigor científico. Núñez Jover particulariza a la ciencia como:

...forma específica de la actividad social y global dirigida a la producción y aplicación de los conocimientos acerca de las leyes objetivas de la naturaleza y la sociedad, aún más, la ciencia se nos presenta como institución social, como sistema de organización científica, cuya estructura y desarrollo se encuentran estrechamente relacionados con la economía, la política, los fenómenos culturales, con las necesidades y las posibilidades de la sociedad. (Núñez Jover, 1999)

\section{Fundamento de la propuesta reflexiva}

El estado que se percibe en el desarrollo de la actividad comunicativa en el entorno de las ciencias hace que se fundamenten procedimientos metodológicos que auguran por la calidad informativa en los contenidos científicos. La búsqueda, selección y clasificación de la información recopilada derivó aplicar el análisis bibliográfico y documental para la evaluación del estado de esta situación problemática del ámbito de la comunicación científica: las revistas científicas desde sus nexos con la responsabilidad social, priorizan sus contenidos para potenciar una eficiente producción científica en función del desarrollo de la sociedad. Este llamado a reflexionar, al cual se convoca en este artículo, es para investigar sobre la problemática: responsabilidad social y su relación con las revistas científicas. Desde una visión generalista se ahonda en criterios, en función de un posible debate científico.

Precisamente la actividad cognitiva, surge como una dimensión de la actividad social protagonizada por personas que contraen compromisos objetivamente condicionados para tratar de comprender y de explicar el movimiento histórico de la ciencia, por lo que plantean los autores, que es también necesario considerar a las relaciones entre la ciencia y la actividad política, ideología, la producción y la sociedad en su conjunto. El actual análisis transdisciplinar que acontece en las ciencias convoca a un estudio profundo que conlleva a procedimientos metodológicos en función de su objetivo principal: el desarrollo de la sociedad.

Desde la base, instituciones y comunidades, la responsabilidad social de las ciencias se manifiesta con acciones y planes propios de acuerdo a sus entornos, que se dirigen a salvaguardar el medio ambiente desde todas sus dimensiones, resultados que potencia la aplicación de la investigación científica, como consecuencia para una comunicación efectiva, gestionada entonces para lograr en los diversos públicos una educación sostenible en pos del desarrollo de sus entornos. 


\section{La comunicación y su visibilidad, prismas de la responsabilidad social}

Constituye la comunicación dentro de las ciencias una disciplina científica de gran importancia, insertada de forma determinante en el desarrollo cada vez mayor del progreso tecnológico y de los cambios socioculturales que se promueven alrededor de estos acontecimientos comunicativos.

Se destaca esta disciplina, la comunicación, por su carácter interdisciplinar, lo que ha motivado a la inexistencia de una definición única, como modelo científico. M. Serrano, (1981) emboca a unas cincuenta definiciones, llegando a la conclusión de que las diferencias entre las mismas estaban motivadas por las disciplinas de las cuales procedían. Desde la visión de Waples precisa este: "es hacer común, compartir, transmitir, preferiblemente interpretado para dos o más personas", sin embargo, Martín Serrano la introduce como:

A todo ser vivo capaz de cambiar información, le conviene el título de "actor de la comunicación". [...] Se infiere que la aptitud para comunicar la posee el hombre y antes que él ya la habían adquirido muchas especies animales, incluso aquellas que están evolutivamente muy alejadas de la especie humana.

Otros autores como George Fierdmann, analizado por M. Serrano,(1981) contempla además que el hecho comunicativo se produce también entre el hombre y la máquina, afirmando que "en las ciencias sociales, toda transmisión de un mensaje entre un emisor y un receptor, es comunicación, ya sea el emisor un hombre o cualquier dispositivo mecánico".

La comunicación científica es un hecho dentro de la responsabilidad de los profesionales de las ciencias por acentuar la sostenibilidad de la sociedad en bien de sus propósitos. La dinámica en la visibilidad y divulgación de las ciencias es un proceso para la aceleración en la implementación de resultados científicos.

Las valoraciones de críticos e investigadores de las ciencias, evidencian que la polémica científica es parte coyuntural del progreso científico; es parte de su validación ante la comunidad científica, un parámetro de su responsabilidad social, así se evidencia en el análisis que se realiza en revistas científicas como parte de sus procesos editoriales. En sus contenidos tenemos a las cartas al editor, formas expresivas, donde los investigadores exponen sus críticas al trabajo publicado por otros, a proyectos en cursos y tendencias disciplinarias entre otras exposiciones, estas acotaciones son consideradas por los editores como las de cualquier otro artículo y son sometidos a una revisión por pares. Esta forma expresiva dentro del discurso científico, apacienta una gran consideración por la comunidad científica, tiene una alta incidencia, e incluso en las estrategias de contenidos de las propias revistas que los publican, al declarar pautas en los estados investigativos que procuran. Evidencian estos contenidos aspectos a tener en cuenta para la implementación de procesos, políticas y líneas de trabajos en contextos sociales, decisorios para el desarrollo de la sociedad. 
Un estudio de Radicchi, precisa en el número de citas recibidas en la base de datos del Web of Science en los artículos de 13 revistas que han recibido "críticas.", a través de esta forma expresiva: carta al Editor" y ejemplifica a Physical Review Letters, con el artículo más citado (20.000 citas) fue un artículo criticado, además asegura que solo el $3 \%$ de los artículos de este tipo han sido comentados, pero a su vez, los 5 artículos más comentados están entre los 16 más citados de esta revista. Lo mismo sucedió, se asevera, en las 12 revistas estudiadas por Radicchi, entre ellas: Nature, Science, Phys. y otras, donde los 5 artículos más criticados siempre están en el top de los más citados. (Radicchi, F, 2012).

\section{La comunicación en las revistas científicas condicionada al interés social}

La personalización de la presentación para comunicar resultados científicos tiene sus específicas determinaciones para el éxito en la comunicación del resultado investigativo. Cada forma expresiva tiene sus pautas que responden a las responsabilidades del canal de comunicación (la revista), ante su comunidad científica y la sociedad en la cual convive, de ahí deviene su connotada responsabilidad social.

El tratamiento ético en los contenidos es estrictamente cuestionando por los consejos científicos y las entidades editoriales, para forjar un proceso en sus contenidos que cumpla con los cánones estipulados, son estos los que implementan las revistas como instrucciones a los colaboradores-autores. Cada disciplina científica dictamina los suyos los cuales responden a las exigencias técnicas y particularidades de las diferentes temáticas científicas que abordan las revistas.

La acción social de las ciencias se condiciona con la responsabilidad de la sociedad y su comunicador (autores y editores), su aceptación ante el conocimiento producido también repercute en la toma de decisiones y en el comportamiento de los sectores industriales, económicos y sociales en general.

La importancia del saber de las ciencias para la sociedad condiciona, transforma y determina el modo de vida y su mejora para la sociedad. El conocimiento de las ciencias es ineludible para el desarrollo; el nivel de percepción que reporta es tal que la concepción de valores se ve cambiada ante la influencia de las tecnologías y su constante desarrollo, la visión reflexiva que propicia los mensajes sobre ciencia es condicional, desde el ámbito de su receptor y las políticas con las cuales convive. Genera el conocimiento y práctica de las ciencias una explicación de la dinámica del mundo actual. (Núñez Jover, 2010)

Al proceso que conlleva la comunicación de las ciencias le caracteriza, ciertas particularidades que debe asumir el editor y los ejecutivos de la revistas científicas, como ente socialmente responsable de los contenidos que se difunde, estos son: (Linares y Santovenia, 2013) 
- Tener presente al método científico y sus procesos, con objetividad, para el tratamiento al hecho, situación problemática o perspectiva abordar;

- Debatir consecuentemente y constructivamente entre lo sucedido, lo conocido y lo posible (pasado-presente-futuro), desde su relación causa-efecto;

- Uso estratégico de herramientas, fuentes y recursos en general que tributen a fundamentar los parámetros éticos y normativos en los se desenvuelve las comunidades y la sociedad;

- Desde la realidad objetiva, tener presente que la investigación es un proceso dinámico, cambiante y continuo;

- El lenguaje exacto en el uso de términos técnicos, de ser posible, explicarlo con oraciones expositivas y argumentativas, apoyándose en el poder de síntesis de la expresión escrita;

- Un propósito: convocar al conocimiento, a la reflexión, el debate y a la continuación de la investigación;

- Asumir con nuestro trabajo una función social que conlleve a las buenas prácticas, mejores formas de vida, con calidad y hacia la sostenibilidad.

La significación de la situación problemática debe reflejarse ante la aplicación de un método del trabajo científico, que será percibido por el lector-usuario en correspondencia con sus intereses de uso. El significado estará exaltado por el tratamiento informativo que le propicie el autor y su editor, donde debe significar para un mejor tratamiento aspectos como:

- Selección eficiente de la información documentaria para develar los significados propuestos dentro de la temática que se investiga;

- Establecer la redacción y estructura del contenido propuesto bajo las instrucciones que estipula la revista;

- Valorar los entornos socio-culturales, geográficos y ambientales entre otras valoraciones.

\section{Las revistas un potencial canal de comunicación científica}

Aseguran Núñez Jover; Montalvo A.; Figaredo C; (2008):

...que el proceso de adquisición de nuevos conocimientos y el desarrollo de las investigaciones fundamentales y aplicadas y las elaboraciones experimentales de diseño son el resultado del trabajo colectivo cada vez más creciente. En este sentido el grupo científico cumple un rol decisivo en la actividad científica....

Las tendencias en la difusión de los resultados científicos, desde la literatura gris que se va propiciando en la comunidad científica (actas de congreso, ponencias, 
artículos, ensayos, monografías, e informes entre otros documentos), se difunde a través de los diferentes canales comunicativos clasificados como: repositorios especializados, científicos, revistas digitales e impresas, y también hasta de forma audiovisual, propósitos comunicativos con objetivos precisos.

A razón de este canal de comunicación, las revistas científicas, "las cuales validan el nuevo conocimiento, lo hacen público y son depositarias de un patrimonio que, siendo intangible, determina la capacidad de progreso de la sociedad", afirma (Krauskopf y Vera, 1995).

Este producto comunicativo ha tenido un gran auge por su crecimiento, desde la aparición ante el público especializado de una primera revista científica en 1663 en Inglaterra. Actualmente existen decenas de miles de revistas científicas en el mundo. En el año 1994, en los comienzos del medio de comunicación masiva Internet, habían en este entorno solo 6 revistas, actualmente son decenas de miles en todo el mundo las que utilizan el ambiente Web para su comunicación científica.

El creciente desarrollo de las publicaciones científicas y en particular las revistas, están marcando modelos de trabajo en la comunicación científica de forma tal que sus niveles de incidencia en la toma de decisiones para el desarrollo, la innovación y la integralidad es cada vez mayor. La coherencia que se precisa para el trabajo en colectivo es cada vez más importante en el logro de la calidad informativa de la actividad científica. Los gestores de contenido que se desarrollan como parte del despliegue de las tecnologías de la información y la comunicación asumen tareas a favor de la coherencia que se impone, destacándose el Open Journal System (OJS) uno de los más generalizado sistemas de gestión editorial, que aboga por la interrelación y el rigor científico a sus contenidos.

En el proceso editorial en el cumplimiento de su agenda temática asegurará los tipos de contribución a instrumentar. Como plantea Guardiola y Saracevik (2005) los artículos de revisión han de cumplir funciones en el desarrollo de la ciencia y la técnica: compactar y sintetizar los conocimientos fragmentados, actualizar e informar sobre el estado de un tema e informar y evaluar la literatura publicada, es su misión, planteamiento que aseveran Cué Brugueras M.; Díaz Alonso G; Díaz Martínez A.; Valdés Abreu M. (2008).

Según Brown (2008), la edición científica se basa en la nueva economía del conocimiento, desde donde le particulariza la apertura, al estar sus contenidos en acceso abierto en un gran porciento; sus metodologías de trabajo están homogenizadas internacionalmente; el poder decisorio está en manos de importantes especialistas nacionales e internacionales; compartir conocimiento es parte de su labor con una gran sentido de responsabilidad como uno de sus pilares.

Las revistas científicas tienen como fin compartir ideas, recursos, proyectos en fin conocimientos es un propósito de la ciencia mundial: Los especialistas utilizan canales formales e informales para divulgar la ciencia global como acreditan los expertos, validan a través de la evaluación por pares, como forma de verificar el conocimiento 
que alimentará los saberes de la comunidad científica internacional. (Torres-Salinas, D. 2008)

Se genera ante este proceso una ruptura de las fronteras disciplinarias y la aparición de las aplicaciones investigativas de carácter inter, pluri y transdisciplinar, como premisa de la comunicación científica. En el contexto Iberoamericano se proyectan normativas de apoyo por parte de la Organización de Estados Iberoamericanos (OEI), las cuales "aseguran la calidad de sus artículos, a fin de que se consoliden como publicaciones de alto impacto internacional, cuya línea editorial (y por consiguiente la línea de investigación que apoyan) estará más inclinada a los temas y enfoques que interesan a los países de Iberoamérica". Como también puntualizan los postulados de la organización estimular la visibilidad a través de plataformas de amplio acceso, es parte de la estrategia de las políticas que se trazan. (http://www.oei.org, 2014)

El puntal en el trabajo por la responsabilidad en la revista lo consttituye la gestión del editor. Su compromiso por fundamentar acciones y aportar razones en las gestión es lo que define la calidad editorial de la publicación. Aseguran investigadores que "la ética del discurso sostiene que los seres humanos, en cuanto seres racionales y razonables, no podemos renunciar a la competencia lingüístico-dialógica sin lesionar nuestra propia dignidad". Según investigadores del marco de la teoría de la corresponsabilidad solidaria en la ética del discurso, la "responsabilidad" es entendida como "capacidad de" y "disposición para" aportar razones con el fin de fundamentar acciones y decisiones frente a todos los demás, y también como "derecho a cuestionar las razones de otros". Es una de las premisas de la labor del editor. (J. Michelini Dorando, 2003)

Las revistas Científica se comprometen con la responsabilidad social que les atañe, como canal comunicativo y vehículo de cohesión de los lineamientos científicos, tecnológicos y medioambientales que estipula la sociedad para su desarrollo. Según P.Simo; García Parra; S. Sunyer y otros autores, (2006), la responsabilidad científica debe concluir beneficiando el desarrollo científico y tecnológico de países de menos desarrollo, siendo así un vehículo conductor de transferencia solidaria de capital intangible.

Las consideraciones hasta aquí formuladas ponen de manifiesto que la ciencia y la tecnología desde el contexto de las revistas científicas son herramientas imprescindibles para impulsar el desarrollo y la cohesión social, son un paradigma que asume la OEI en los países de Iberoamérica. Desde un determinante contexto científico: el universitario, se especifica a razón de los criterios de Vázquez Alonso, A. ; Manasero Mas, M. (2012), que la profunda interacción entre ciencia y tecnología en la actividad científica de la actualidad, también identificada como, tecnociencia, traduce una nueva visión de la educación científica donde se integra a la tecnología. El slogan de "alfabetización científica y tecnológica para todos", como logro de la necesaria educación y competencia científica de los estudiantes, estos, base en la formación profesional y científica del ente social, es un pilar del componente innovador en la alfabetización para todos. 


\section{Como conclusiones}

- Las ciencias avanza con el devenir histórico y se desarrollan afín a los objetivos de la sociedad; sus mecanismos de comunicación se alinean a las comunidades científicas a través de las revistas científicas como vehículo comunicacional.

- La responsabilidad social es un paradigma para el desarrollo, donde las revistas científicas a través de la gestión editorial que protagonizan sus editores, autores e investigadores, asumen una responsabilidad social decisoria para el desarrollo de la sociedad, desde donde las tecnología se adjudican un importante protagónico con ética y probado rigor científico; por lo que la comunicación de los resultados de investigación científica por este canal comunicativo, se asume como vía para contribuir a la sostenibilidad de la sociedad.

- Las revistas son productos comunicativos de gran impacto, los cuales se difunden a través de canales impresos y digitales, e incluso ya se crecen también en el medio audiovisual, establecen estrategias que validan el nuevo conocimiento, lo hacen público y son depositarias de un patrimonio que, siendo intangible, determina la capacidad de progreso de la sociedad.

\section{Bibliografía}

Briggs, Mark.(2007). Journalism 2.0: How to Survive and Thrive: A digital literacy guide for the information age. Disponible en: http://www.kcnn.org/resources/journalism_20 /22/11/2013]

Brown, David J.; Boulderstone, Richard. (2008). The impact of electronic publishing: the future for publishers and Librarians. Munich: Saur. Disponible en: http:// sciencie.readComt.org.

Cué Brugueras M.; Díaz Alonso G; Díaz Martínez A.; Valdés Abreu M. (2008). El artículo de revisión. Revista Cubana de Salud Pública, versión On-line ISSN 0864-3466.Disponible en: http://scielo.sld.cu/scielo.php?script=sci_arttext\&pi$\mathrm{d}=\mathrm{S} 0864-34662008000400011$ [22/11/2013]

Delgado López-Cózar, Emilio; Ruiz Pérez, Rafael; Jiménez Contreras, Evaristo. (2007). La Edición de Revistas Científicas: Directrices, Criterios y Modelos de Evaluación. Madrid: Fundación Española para la Ciencia y la Tecnología.

Grávalos Macho, David (2012). Uso y explotación eficientes de una página web como herramienta de comunicación para empresas y organizaciones: decisiones estratégicas y tácticas. Caldevilla Domínguez; David (compilador) Capítulo IX Nuevas Tendencias en Investigaciones sobre. Comunicación en el EEES. Universidad Europea de Madrid, Editorial, Visión Libros, Madrid, España. pp.159-16

Gutiérrez-Cuevas, C. (2001). Responsabilidad social de la empresa en la sociedad de la información, Taller Trasandino de Responsabilidad Social Empresarial -RSE-, Lima, Perú 
http://www.scielo.cl/scielo.php?pid=S071658112003001400015\&script=sci_arttext\&tlng=ptLiteraturaylingüística.pdf[22/11/2013]

J. Michelini Dorando, (2003) La ética del discurso como ética de la responsabilidad. Revista Literatura y Lingüística ${ }^{\circ} 14$, Chile. Disponible en:

Krauskopf, Manuel y Vera, María Inés;(1995). Las revistas latinoamericanas de corriente principal: indicadores y estrategias para su consolidación. Revista científica Interciencia 20(3): 144-148. Url: http://www.interciencia.org.ve [22/11/ 2012]

Linares H.M.P. Procedimientos y Valoraciones en la gestión editorial desde el gestor Open Journal System: revista Ciencias de la Información, revista digital de Comunicación Razón y Palabra, edición No.. 89/2014, disponible en: http://www. razonypalabra.org [22/1/ 2014]

M. Alejandra Rojas V.; Sandra Rivera M. (2010). Guía de Buenas Prácticas para Revistas Académicas de Acceso Abierto. Disponible en: http://www.revistasabiertas.com; y en http://www.derechosdigitales.org [22/11/2012]

Martín Serrano, M., et al. (1981).Epistemología de la comunicación y análisis de la referencia. Ed. Cuadernos de la Comunicación. Vol.VIII. Madrid, España

Medford Cárdenas M,Ordoñez Povea B,Garzó Rueda RL, Carrazana García D. (2010). Ética en la ciencia y tecnología. Un enfoque desde la educación médica superior. Rev méd electrón[Seriada en línea] 2010;32(1). Disponible en URL: http://www.revmatanzas.sld.cu/revista \%20medica/ano\%202010/vol6\%202010/ tema08.htm [22/11/2012]

Mendelsohn, E (1977): "The social construction of scientific knowledge", Mendelsohn, et.al, (comps), The social production of scientific knowledge, Reidel Publishing Company, Dordrecht y Boston. Citado por (Nuñez Jover, Jorge; 1999) Rigor, objetividad y responsabilidad social: la ciencia en el encuentro entre ética y epistemología, pág. 77 .

Metzeltin, Miguel, (2002) De la retórica al análisis del discurso revista electrónica de estudios filológicos de la retórica al análisis del discurso, Universidad de Viena / Academia Austríaca de Ciencias) Metzeltin, Miguel / Thir, Margit, El arte de contar: una iniciación, Universidad, Murcia, España.

Nuñez Jover, J.; Montalvo Arriete, Luis F.; Figaredo Curriel F.; (2008) Pensar, Ciencia, Tecnología y Sociedad. Editorial Félix Varela, La Habana, Cuba 119 -128 y 153

Núñez Jover, Jorge, (2010).Conocimiento académico y Sociedad. Ensayos sobre política universitaria investigación y posgrado, editorial UH, La Habana, Cuba.

P. Simo; M.García Parra; S. Sunyer G. Trullas; M. Jorda; (2006). Hacia la evolución de la calidad, el prestigio y la responsabilidad social de las revistas de Acceso Abierto. Revista Intangible Capital, enero-marzo, año.vol. 2 no. 001, Barcelona, España pp.1-20

Proyecto 2021; (2014). Página inicial del sitio. http://www.oei.org consultado [22-112013] 
Radicchi, Filippo (2012). In science $\backslash$ there is no bad publicity": Papers criticized in technical comments have high scienti_c impact., edición, 22 Sep 2012 http:// physics.org/arXiv:1209.4997v.soc-ph Consultado [22-11-2012]

Torres- Salinas, D. (2008). La edición y las revistas científicas ante la encrucijada 2.0. Disponible en: http://www.thinkepi.net/la-edicion-y-las-revistas-cientificas-ante-la-encru[18-1-2013]

Torres-Salinas, Daniel. (2008b) ¿Qué es y cómo se edita una revista científica 2.0?. En: VIII Semana de la Ciencia. Mesa de Debate: Las revistas científicas 2.0 ¿Existen? Madrid, 11 de noviembre. Disponible en: http://ec3noticias.blogspot. com/2008/11/qu-es-y-cmo-se-edita-una-revista-20.html [22/11/2012]

Vázquez Alonso, A. ; Manasero Mas, M. (2012). La selección de contenidos para enseñar naturaleza de la ciencia y tecnología (parte 1): Una revisión de las aportaciones de la investigación didáctica Revista Eureka sobre Enseñanza y Divulgación de las Ciencias. 2012, Vol. 9 Issue 1, p2-31. 30p. Disponible en: http:// web.a.ebscohost.com/ehost/detail?sid=3f6cb020-3b28-4b7b-b068-7a285992338 9\%40sessionmgr4004\&vid=1\&hid=4209\&bdata $=$ Jmxhbmc9ZXMmc210ZT1laG9zdC1saXZl\#db=aph\&AN=73876843 Consultado [12-11-2012] 